\title{
ANÁLISE PALEOPALINOLÓGICA DO ALBIANO NA BACIA DE CAMPOS
}

\author{
Clara Rodrigues NASCIMENTO \\ José Alexandre de Jesus PERINOTTO \\ Mitsuru ARAI
}

\begin{abstract}
RESUMO
O presente trabalho teve como objetivo identificar os conjuntos palinológicos marinhos e continentais da Bacia de Campos e analisar a sua distribuição ao longo das seções geológicas, a fim de testar modelos paleoambientais e paleobiogeográficos. O material palinológico estudado é proveniente dos poços Bonito e Pampo, Albiano, Grupo Macaé. Os grãos de pólen gimnospérmicos Classopollis classoides, Gnetaceapollenites spp. e Equisetosporites spp., todos de caráter xeromórfico, têm alta frequência no material analisado. As espécies de dinoflagelados mais abundantes foram as cosmopolitas Spiniferites ramosus, Odontochitina operculata e Trichodinium castanea. Quatro espécies de dinoflagelados tipicamente tetianas (Codoniella campanulata, Cyclonephelium vannophorum, Endoceratium dettmanniae e Tehamadinium mazaganense) foram identificadas. Constatou-se aumento na proporção de elementos continentais nos estratos correspondentes ao final do Albiano, concomitantemente à passagem de uma litologia carbonática para uma outra composta por folhelhos e margas. Houve aumento na riqueza de palinomorfos marinhos - da base para o topo - nas seções estudadas, passando de uma biota com baixa diversidade - dominada por palinoforaminíferos - para outra biota mais diversa e com diferentes espécies de dinoflagelados. A integração dos dados micropaleontológicos permite inferir que durante o estabelecimento do Oceano Atlântico Sul a Bacia de Campos recebeu águas provenientes do Mar de Tétis, fato que refuta o modelo clássico da entrada de águas austrais acompanhando a abertura tectônica de sul para norte.
\end{abstract}

Palavras-chave: palinologia, Bacia de Campos, dinoflagelados, Albiano.

\section{ABSTRACT}

PALEOPALYNOLOGICAL ANALYSIS OF THE ALBIAN IN THE CAMPOS BASIN. The present work was aimed to identify the marine and terrestrial palynomorphs from the Campos Basin and to analyze their distribution in geological sections in order to evaluate paleoenvironmental and paleobiogeographic models. The palynological material comes from the Bonito and Pampo wells, Albian, Macaé Group. Gymnospermous pollen grains occur in considerable abundance, especially Classopolis classoides, Gnetaceapollenites spp. and Equisetosporites spp., which are typically xeromorphic. The most abundant dinoflagellates are the cosmopolitan species Spiniferites ramosus, Odontochitina operculata and Trichodinium castanea. Four typically Tethyan dinoflagellate species (Codoniella campanulata, Cyclonephelium vannophorum, Endoceratium dettmanniae and Tehamadinium mazaganense) were identified. The proportion of land-derived palynomorphs increases in the strata at the end of the Albian, concomitant with the transition from carbonates to shales and marls. Furthermore, marine palynomorphs increase in diversity from the base to the top of the studied sections, changing from a biota dominated by palynoforaminifera to one with high diversity and rich in dinoflagellates. Integration of the micropaleontological data indicates that, during the formation of the South Atlantic Ocean, the Campos Basin received water from the Tethys Ocean, which contradicts the classic model of Austral water incursion simultaneously with tectonic opening from south to north.

Keywords: palynology, Campos Basin, dinoflagellates, Albian. 


\section{INTRODUÇÃO}

O presente trabalho constitui uma contribuição à Palinologia da seção albiana da Bacia de Campos, onde, em função da intensa atividade exploratória de petróleo iniciada na década de 1970 , vem se acumulando informações geológicas importantes que contribuem para o entendimento do processo de evolução do Oceano Atlântico Sul. Até o Eocretáceo os continentes sulamericano e africano encontravam-se unidos constituindo o supercontinente Gondwana; há aproximadamente 125,5 milhões de anos, iniciou-se sua separação (LARSON \& LADD 1973). Existem duas principais hipóteses, que ainda são motivo de discussões e polêmicas no meio científico, para explicar o processo de estabelecimento do Oceano Atlântico Sul e sua conexão com o Atlântico Norte/Central. O modelo clássico, que vinha sendo aceito tradicionalmente, defende a entrada das águas pelo sul, acompanhando o processo de abertura tectônica que separou os dois continentes (OJEDA 1982; DIAS 2005, 2008). Entretanto, dados paleontológicos sugerem que as primeiras incursões foram constituídas pelas águas provindas do norte, em função da afinidade das biotas aptiana e albiana das bacias da margem leste brasileira com aquela biota presente no Mar de Tétis - semelhanças entre grupos de amonites, foraminíferos planctônicos, pitonelídeos, dinoflagelados (KENNEDY \& COOPER 1975, WIEDMAN \& NEUGEBAUER 1978, KOUTSOUKOS \& DIAS-BRITO 1987, DIAS-BRITO 1987, ARAI et al. 1994, DIAS-BRITO 2000, BENGTSON et al. 2007, ARAI 2009).

Assim, esse artigo visa compreender como os palinomorfos distribuem-se ao longo da seção geológica do Grupo Macaé da Bacia de Campos, a fim de agregar os conhecimentos ecológico e paleobiogeográfico ao panorama científico vigente sobre o tema. Para se atingir esses objetivos foi necessário: 1) realizar um levantamento das espécies de microfósseis que ocorrem nos poços Bonito e Pampo da Bacia de Campos, em estratos referentes ao Albiano (Cretáceo Inferior); 2) comparar quantitativamente as ocorrências de palinomorfos continentais e marinhos a fim de testar modelos paleoambientais; 3 ) elaborar gráficos e analisar a distribuição dos palinomorfos ao longo das seções dos poços; 4) comparar a associação de dinoflagelados identificada com as associações equatoriais e austrais, a fim de discorrer sobre modelos paleobiogeográficos de instalação do Oceano Atlântico Sul.

\section{2 ÁREA DE ESTUDO}

A Bacia de Campos situa-se na margem continental do Brasil e abrange a costa sul do estado do Espírito Santo até a costa norte do estado do Rio de Janeiro, entre os paralelos $21^{\circ}$ e $23^{\circ}$ sul. Seu limite norte, com a Bacia do Espírito Santo, corresponde ao Alto de Vitória, enquanto o sul, com a Bacia de Santos, é demarcado pelo Alto de Cabo Frio (Figura 1). A sua área de abrangência corresponde a $100.000 \mathrm{~km}^{2}$, dos quais $500 \mathrm{~km}^{2}$ estão em área emersa (RANGEL et al. 1994). Os poços aqui estudados foram os de Bonito (BO) e Pampo (PM).

O pacote sedimentar diagnóstico da instalação do Atlântico Sul na Bacia de Campos corresponde ao Grupo Macaé, uma vez que esse é portador dos primeiros elementos marinhos da bacia. $\mathrm{O}$ termo Macaé foi utilizado inicialmente como Formação por SCHALLER (1973) para se referir aos sedimentos depositados sobre os evaporitos da bacia; posteriormente, a unidade foi reclassificada como Grupo por WINTER et al. (2007). Calcarenitos e calcilutitos correspondem à base da sequência, enquanto a parte superior é composta por calcilutitos, margas e folhelhos (DIAS-BRITO 1982). O Grupo Macaé foi a primeira unidade litoestratigráfica offshore a ser reconhecida como portadora de petróleo comercial na Bacia de Campos nos anos 1970 (ARAI \& LANA 2004) e, a partir daí, ela se o que a tornou objeto de muitos estudos a partir de então.

\section{MATERIAIS E MÉTODOS}

O material estudado consistiu em lâminas confeccionadas no CENPES - Centro de Pesquisas \& Desenvolvimento Leopoldo Américo Miguez de Mello (Petrobras, Rio de Janeiro - RJ), seguindo o processo de preparação de material palinológico descrito por UESUGUI (1979). É oportuno registrar que estas lâminas são duplicatas montadas pelo pesquisador Mitsuru Arai e não pertencem ao acervo oficial do CENPES, e seu processo de tombamento junto ao UNESPetro - Centro de Geociências Aplicadas ao Petróleo encontra-se em andamento. A pesquisa enfocou o estrato geológico referente ao andar Albiano (Série Cretáceo Inferior). No poço Bonito, foram amostrados 21 níveis, enquanto no poço Pampo, 14 níveis; para cada nível foram confeccionadas 3 lâminas. Essas lâminas estão identificadas na coleção em ordem crescente de profundidade e as letras seguidas dos números indicam as duplicatas. Dessa forma, a sigla BO-1A indica uma lâmina do poço Bonito, referente ao primeiro nível ou profundidade amostrada, enquanto 


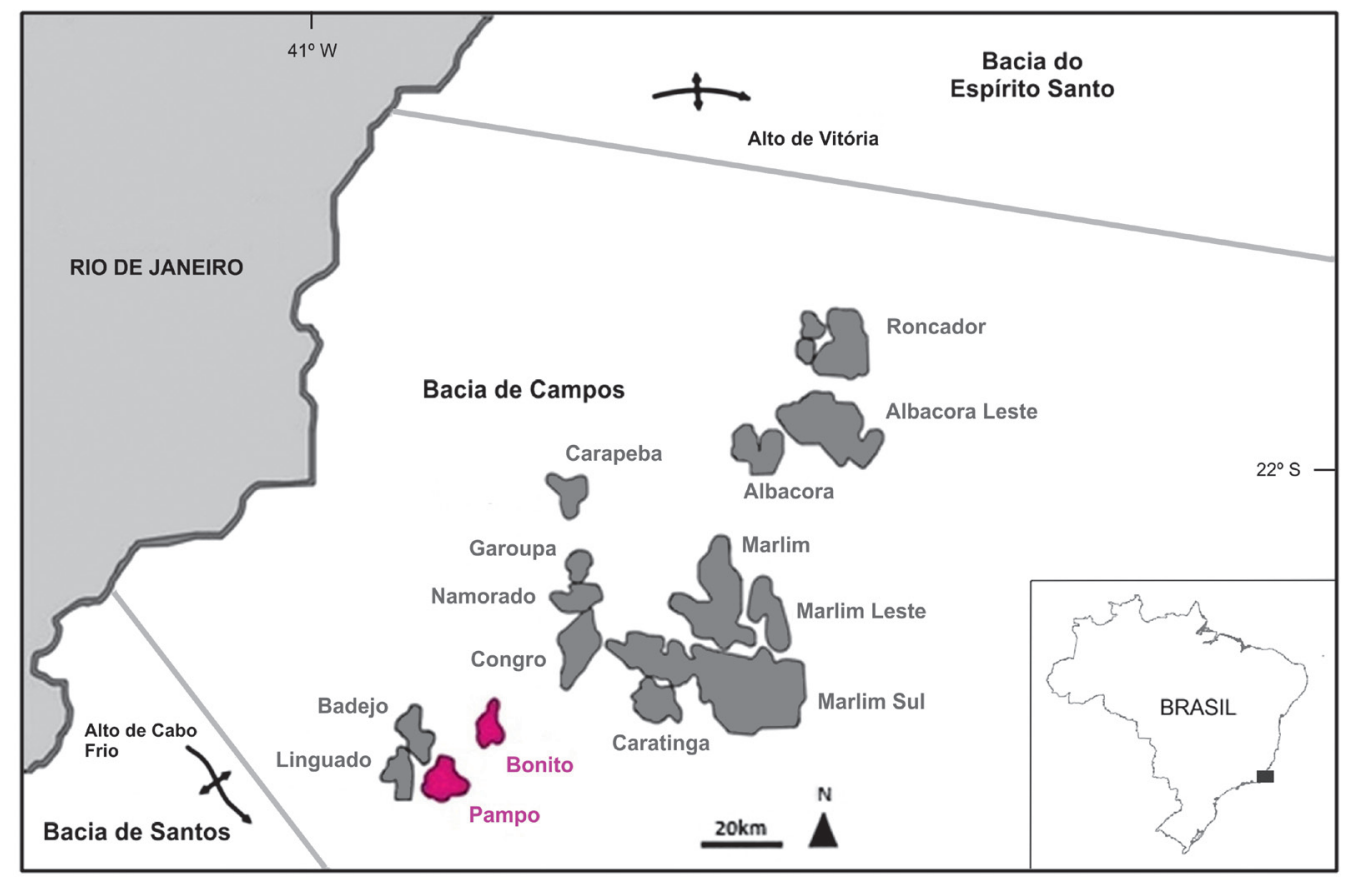

FIGURA 1 - Localização da Bacia de Campos. Destaque (em rosa) para os campos petrolíferos Bonito e Pampo, onde se situam os poços estudados.

a PM-3B corresponde a uma lâmina do poço Pampo, referente à terceira profundidade amostrada.

A análise das lâminas foi realizada no microscópio óptico Leica, pertencente ao Laboratório de Bioestratigrafia e Fotomicrografia do UNESPetro, acoplado a uma objetiva de 20x para varredura de toda sua área, com aumento de 200x. Os dados quantitativos foram reunidos numa planilha Excel e, posteriormente, juntadas às tabelas de distribuição dos palinomorfos em função da profundidade dos poços. Utilizou-se o programa Leica Application Suite 4 para a obtenção das fotomicrografias dos palinomorfos.

Os gráficos de distribuição dos palinomorfos versus profundidade dos poços foram executados com os programas PAST (PAleontological STatistics, versão 1.49) e C2 Data Analysis, versão 1.7.5 (HAMMER et al. 2001, JUGGINS 2007).

A análise qualitativa consistiu na identificação dos palinomorfos e na sua classificação em dois grupos: continentais e marinhos. Quando possível, a identificação foi efetuada até o nível de espécie baseando-se na consulta a artigos especializados (DINO et al. 1999, TORRICELLI 2000, LENTIN \& WILLIAMS 2004, OOSTING et al. 2006), bem como a uma base de dados online sobre dinoflagelados fósseis e viventes, a DINOFLAJ2 (FENSOME et al. 2008).

A contagem da frequência dos palinomorfos ocorreu de forma direta, ou seja, todo palinomorfo encontrado nas lâminas era contabilizado.

\section{RESULTADOS}

Ao todo, 12 táxons continentais e 81 táxons marinhos foram identificados. O Apêndice apresenta a listagem de todos os palinomorfos, classificados em grandes grupos (esporos, grãos de pólen, dinoflagelados e demais palinomorfos marinhos).

Dentro do grupo dos palinomorfos continentais, os grãos de pólen gimnospérmicos Classopolis classoides (Figuras 2D, E) e Gnetaceaepollenites spp. (Figuras 2L, M) foram os mais representativos em ambos os poços, seguidos por Equisetosporites spp. (Figuras 2J, K). Apenas um gênero de esporo de pteridófita, Cicatricosisporites sp, foi encontrado. (Figura 2A). Os grãos de pólen elaterados são representados principalmente pelas espécies Elaterosporites klaszi (Figura 2G), Elaterosporites protensus (Figura 2H) e Elaterosporites verrucatus (Figura 2I).

Dentre os palinomorfos marinhos (Figuras 3 e 4), os cistos de dinoflagelados formam, sem dúvida, o grupo mais significativo, tanto em riqueza como em abundância, representando quase a totalidade dos microfósseis marinhos, com 78 espécies identificadas agrupadas em 49 gêneros. Por outro lado, os palinoforaminíferos (Figura 4L) foram o segundo grupo mais representativo.

No grupo dos dinoflagelados, a espécie Spiniferites ramosus (Figura $3 \mathrm{~J}$ ) foi a mais frequente 

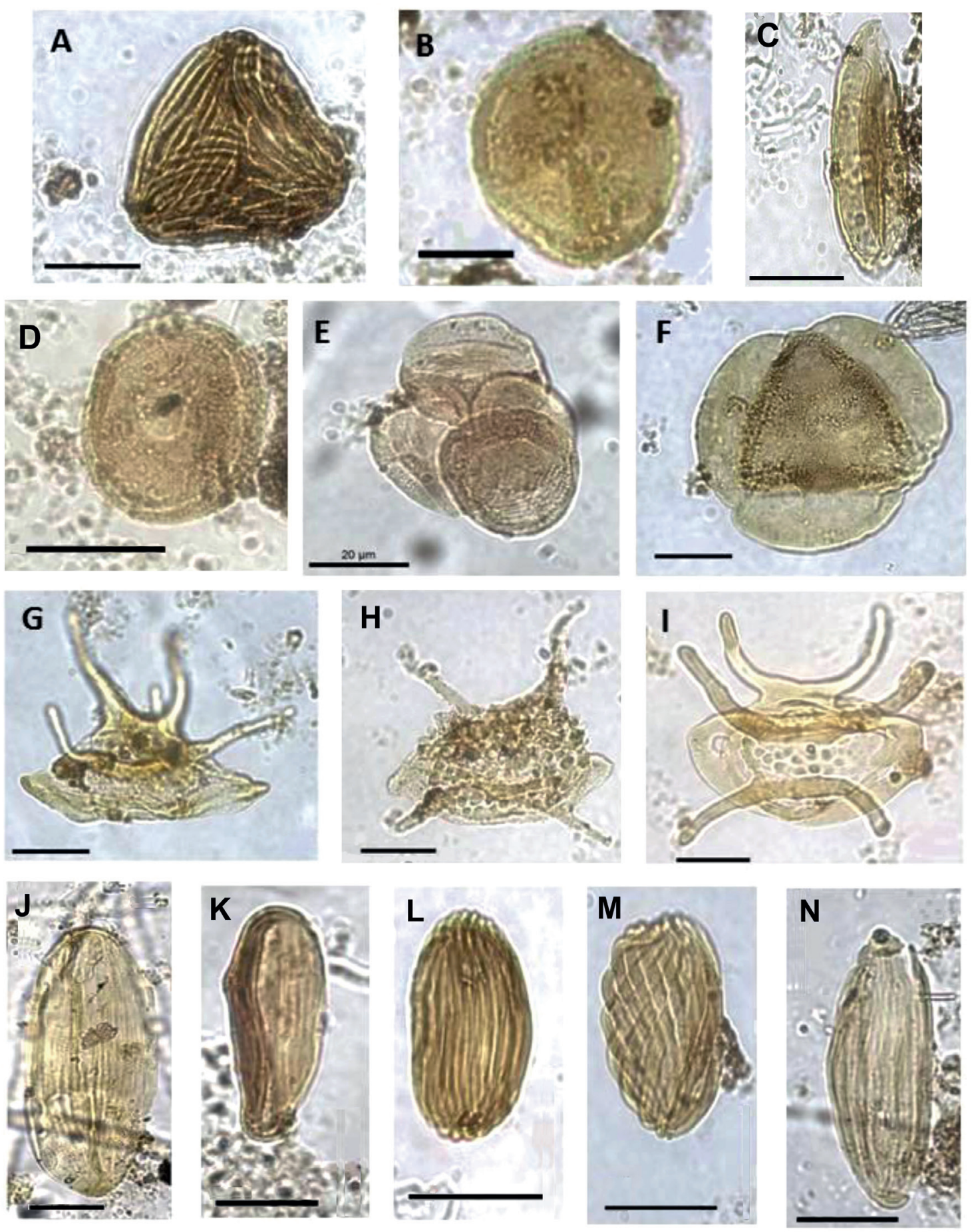

FIGURA 2 - Fotomicrografias dos palinomorfos continentais. Todas as escalas são de $20 \mu \mathrm{m}$. A. Cicatricosisporites sp. BO 10-A (34,9; 82,1). B. Circulina sp. BO 13-C $(20,8 ; 93,9)$. C. Cycadopites sp. BO 7-B $(42,5 ; 73,9)$. D. Classopollis classoides. BO 5-A $(15,5 ; 89,9)$. E. Tétrade de Classopollis classoides. BO 6-B $(38,7 ; 76,5)$. F. Callialasporites trilobatus. PM 8-D $(40,5 ; 65,1)$. G. Elaterosporites klaszii. PM 10-C $(31,6 ; 18,9)$. H. Elaterosporites protensus. PM 6-C $(12,1 ; 86,5)$. I. Elaterosporites verrucatus. PM 8-E $(24,3$; 71,2). J, K. Equisetosporites spp. BO 4-A (34,2; 80,4). L, M. Gnetaceaepollenites spp. BO 20-B $(20,5 ; 78,4)$. N. Steevesipollenites sp. BO 7-A $(33,3 ; 69,1)$. 

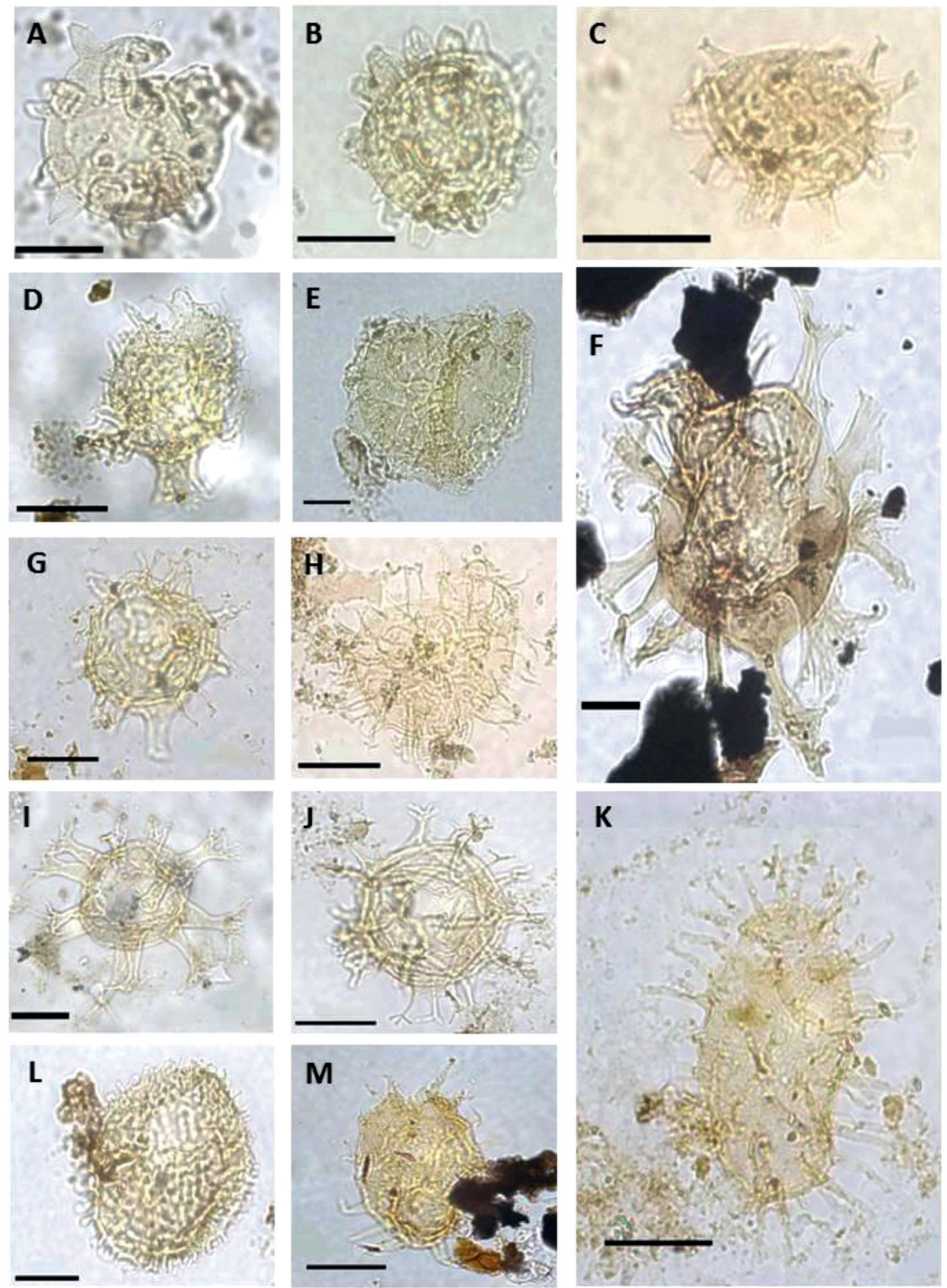

FIGURA 3 - Fotomicrografias dos dinoflagelados. Todas as escalas são de $20 \mu \mathrm{m}$. A, B. Litosphaeridium conispinum. BO 5-B $(35,4 ; 87,7)$. BO 6-A $(15,2 ; 78,9)$. C. Litosphaeridium sp. BO 13-E $(32,0 ; 15,8)$. D. Coronifera oceanica. PM 7-C $(29,8 ; 57,2)$. E. Cyclonephelium vannophorum. PM 2-A (25,1; 69,2). F. Cordosphaeridium sp. PM 3-A (42,2; 78,5). G. Florentinia sp. PM 6-C (51,3; 27,8). H. Impletosphaeridium sp. BO 5-B (20,7; 74,5). I. Oligosphaeridium itajaiensis. BO 3-A $(38,2 ; 73,6)$. J. Spiniferites ramosus. PM 8-C $(41,3 ; 57,1)$. K. Tanyosphaeridium sp. BO 13-A $(31,8 ; 88,8)$. L. Trichodinium castanea. PM 4-C (23,7; 80,2). M. Prolixosphaeridium sp. PM 8-D (47,1; 29,8). 

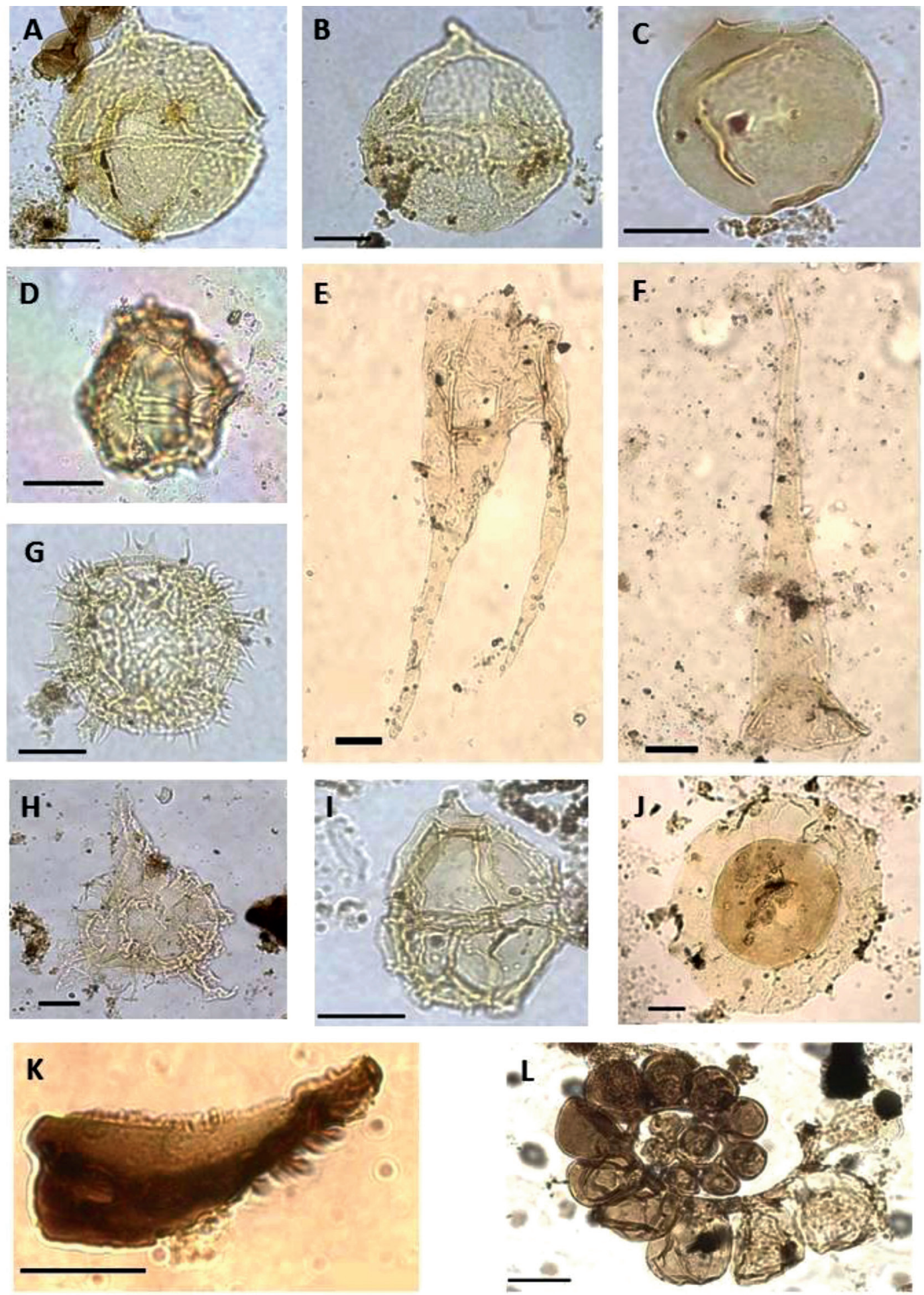

FIGURA 4 - Fotomicrografias dos dinoflagelados e outros palinomorfos marinhos. Todas as escalas são de $20 \mu \mathrm{m}$. A, B. Cribroperidinium sp. PM 8-A (19,5; 90,7). PM 4-B $(39,5 ; 71,2)$. C. Fromea amphora. BO 3-A $(27,1 ; 58,6)$. D. Leptodinium sp. PM 11-B (29,8; 33,5). E. Odontochitina operculata. Cisto sem opérculo. BO 3-A (17,9; 41,3). F. Odontochitina operculata. Opérculo. BO 3-A $(33,5 ; 72,6)$. G. Pervosphaeridium cenomaniense. PM 3-B (34,5; 62,4). H. Pseudoceratium sp. PM 3-B (34,3; 73,2). I. Wrevittia helicoidea. BO 7-A (29,7; 75,0). J. Pterospermopsis sp. BO 6-A (40,8; 88,6). K. Escolecodonte. PM 3-B (28,7; 47,3). L. Palinoforaminífero espiral. BO 10-A (25,4; 90,9$).$ 


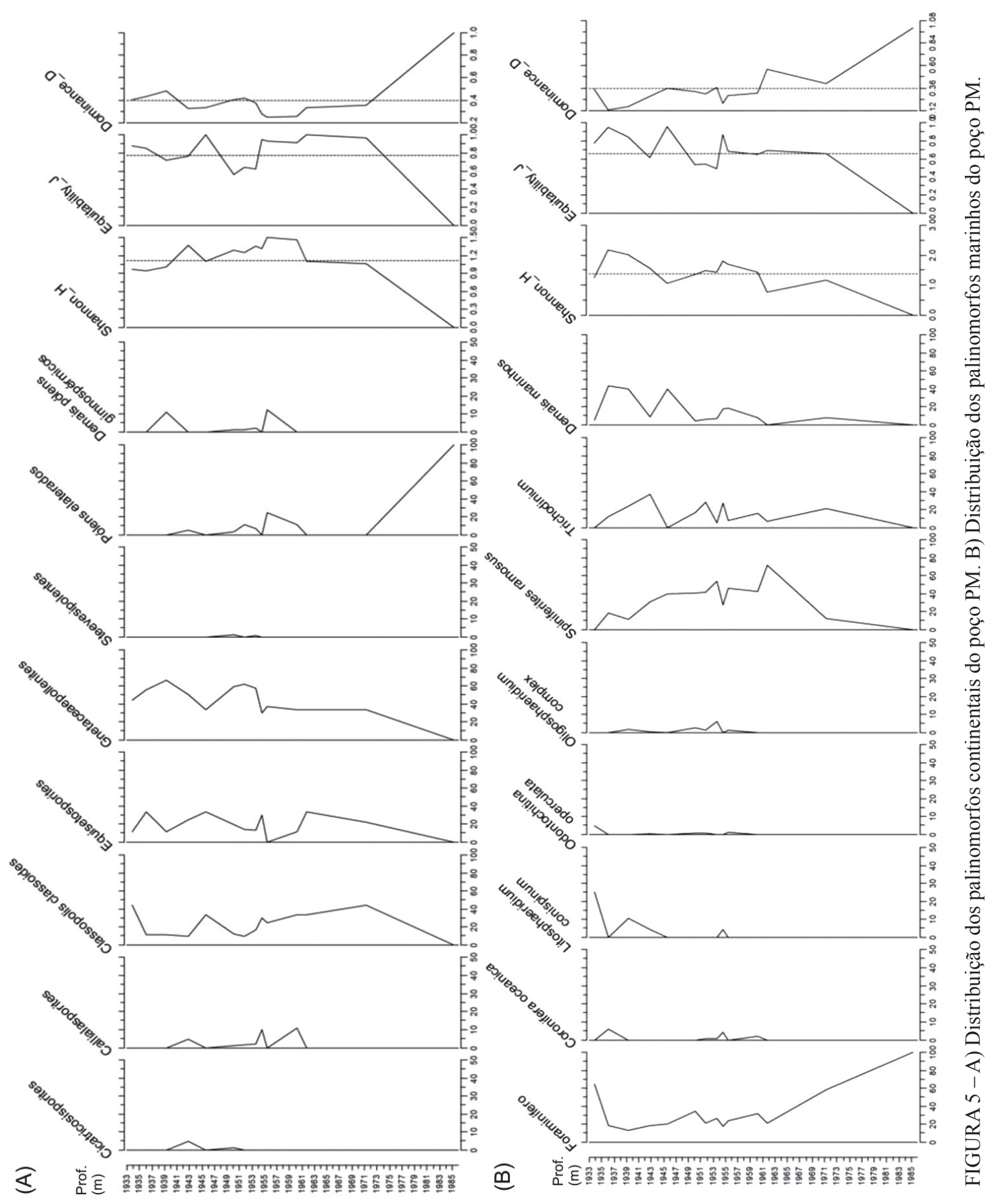


em ambos os poços, seguida pela Trichodinium castanea (Figura 3L), Litosphaeridium conispinum (Figuras 3A, B), Oligosphaeridium complex e Odontochitina operculata (Figuras 4E, F).

Com relação às espécies-guia de dinoflagelados, destacam-se a espécie Litosphaeridium conispinum (Figuras 3A, B), exclusiva do Albiano superior (COSTA \& DAVEY 1992), e a Litosphaeridium arundum, restrita a todo o Albiano (DAVEY 1979, WILLIAMS \& BUJAK

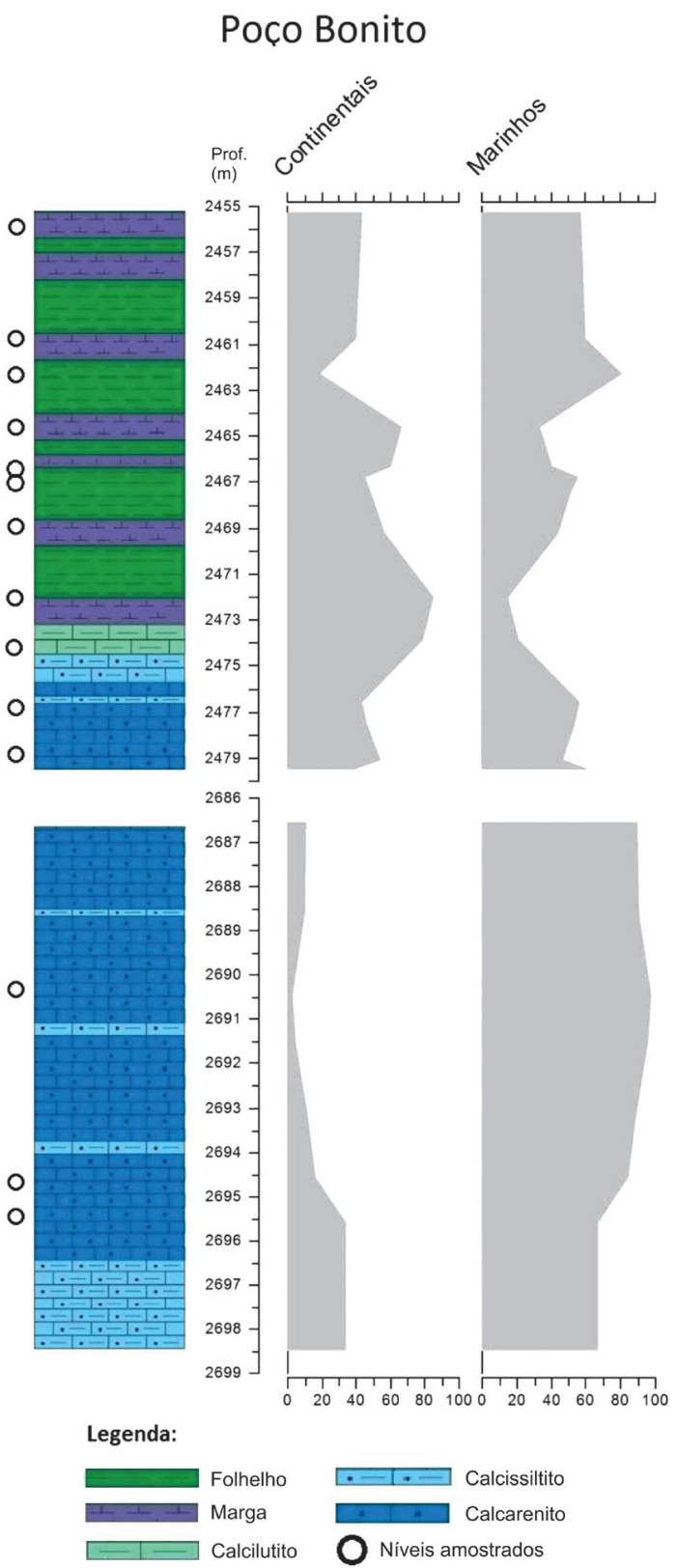

FIGURA 6 - Perfil litológico, níveis amostrados e porcentagem de palinomorfos marinhos e continentais ao longo do poço Bonito.
1985). Ambas as espécies já foram registradas em estratos albianos da Austrália, Espanha, França, Itália, Egito, Congo e da Califórnia e são usadas como marco delimitador da passagem do Albiano para o Cenomaniano (AURISANO 1992).

A distribuição dos palinomorfos versus profundidades dos poços permite identificar os táxons com maior ou menor abundância, bem como os níveis onde ocorrem os eventos de dominância (Figura 5).

Ao se comparar a incidência de palinomorfos continentais e marinhos e suas respectivas distribuições ao longo das seções, observou-se que ocorre um incremento dos elementos continentais da base para o topo (Figuras 6 e 7). O aumento de palinomorfos continentais é mais notável na seção superior do poço $\mathrm{BO}$ e mais discreto no poço $\mathrm{PM}$, o qual apresentou maior porcentagem de palinomorfos marinhos ao longo de toda sua seção.

Na seção superior do poço BO, os palinomorfos continentais e marinhos apresentam frequências semelhantes $-51,8 \%$ e $48,2 \%$, respectivamente.

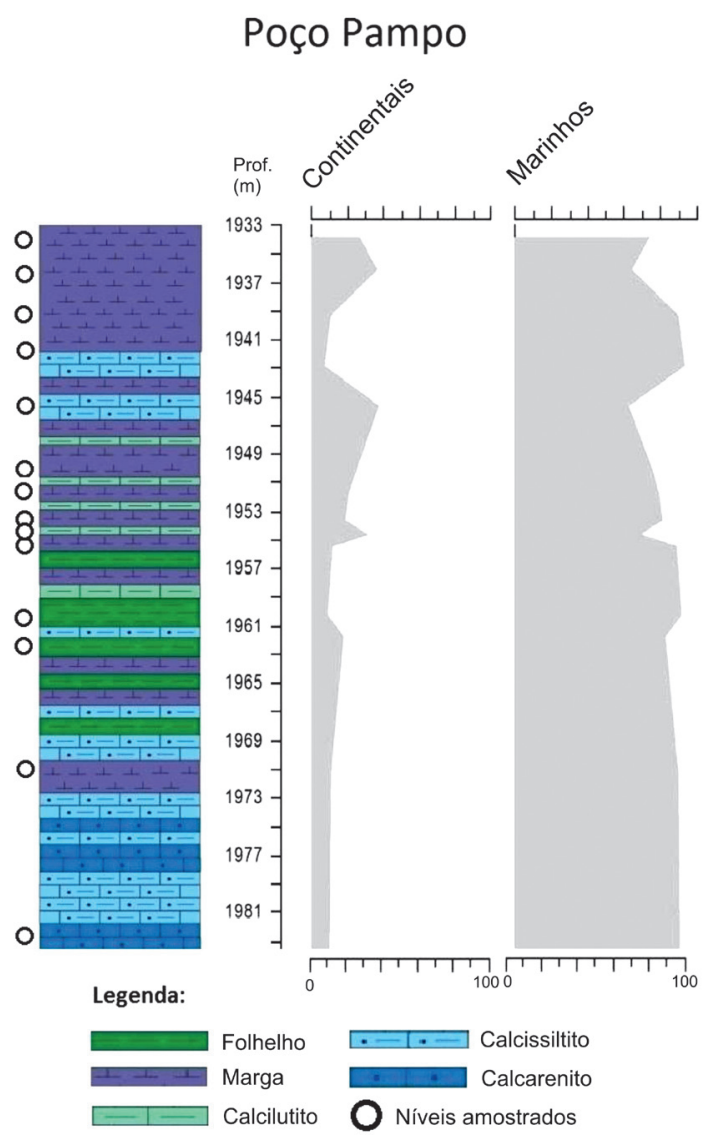

FIGURA 7 - Perfil litológico, níveis amostrados e porcentagem de palinomorfos marinhos e continentais ao longo do poço Pampo. 
$\mathrm{Na}$ seção inferior, contudo, essa proporção muda abruptamente: a frequência de palinomorfos continentais cai para $15,1 \%$ e a frequência de palinomorfos marinhos sobe para $84,9 \%$. No poço PM a mudança nos valores de frequência dos palinomorfos continentais não é tão evidente como no poço $\mathrm{BO}$, mas é possível notar um acréscimo nas suas porcentagens da base para o topo da seção de $11 \%$ para $19 \%$, com picos de $37 \%$.

\section{DISCUSSÃO}

Uma hipótese possível para explicar o aumento de palinomorfos continentais em ambos os poços seria a ocorrência de mudança climática com acréscimo de umidade no local. Com maior precipitação o aporte de material terrígeno para dentro da bacia aumentaria, da mesma forma que a proporção de esporos e grãos de pólens. No entanto, o caráter da assembleia de palinomorfos continentais não permite sustentar essa teoria. Os grãos de pólen e esporos encontrados são diagnósticos de aridez e se fazem presentes nas amostras em porcentagens relativamente altas, como Classopollis classoides, Equisetosporites sp. e Gnetaceaepollenites sp. Até mesmo Callialasporites sp. e o esporo Cicatricosisporites sp., presentes em pequena quantidade, são característicos de climas relativamente secos.

Outra hipótese possível, proposta por DIASBRITO (1995), seria a oscilação no nível do mar. $\mathrm{O}$ autor integrou os dados de zoneamento publicados por ARAI \& UESUGUI (1989) a novos dados paleoclimáticos baseados em associações de foraminíferos calcários. De acordo com o autor, a Bacia de Campos passou por uma fase marcante de alterações hidrológicas na transição Mesoalbiano Neoalbiano. Até o Mesoalbiano a sedimentação da bacia ocorria em grande ecossistema de rampa carbonática, em águas quentes, hipersalinas e ricas em $\mathrm{CaCO}_{3}$. $\mathrm{O}$ influxo de terrígenos à bacia era bastante baixo. Essa fase foi batizada de $\alpha 1$, cujo intervalo correspondente foi considerado como Albiano inferior a médio. Após esse momento iniciou-se a fase $\beta 0$ (Neoalbiano), marcada por uma elevação no nível do mar e início do afogamento da rampa carbonática com manutenção do clima árido. Com a subida eustática, mais áreas continentais foram alcançadas, trazendo material terrígeno para dentro da bacia. Dessa forma, as águas, até então límpidas e pobres em nutrientes, tornaram-se férteis. Com a contínua elevação do nível do mar, a bacia passou por um momento de instabilidade e ciclos de ressurgência, o que determinou a deposição de ritmitos e culminou com o afogamento da rampa carbonática e, conse- quentemente, o fim da deposição calcária na bacia. Esse último ciclo corresponde à fase $\beta 1$. $\mathrm{O}$ aumento na proporção de elementos continentais nos poços (da base para o topo) poderia ser explicado, então, pelo início da fase $\beta 0$ na Bacia de Campos.

A passagem do Albiano médio para superior ficou registrada também na diversidade da associação palinológica da Bacia de Campos. É possível observar que a riqueza aumenta da base para o topo das seções estudadas (Figura 8), o que vai ao encontro do postulado por ARAI et al. (2000) e ARAI (2009), ao afirmarem que a diversidade na assembleia de dinoflagelados das bacias de Campos e Santos atinge valores elevados no Vraconiano. A interpretação para o observado seria o desaparecimento da barreira física existente alinhamento entre Dorsal de São Paulo e Alto de Florianópolis ou Microcontinente Rio Grande - e a entrada de águas austrais.

O mesmo aumento de diversidade já havia sido observado por DIAS-BRITO (1995) nas associações de foraminíferos bentônicos e planctônicos das bacias da margem continental brasileira durante a transição citada, fato que o autor atribuiu à elevação do nível do mar (passagem de mar restrito para oceano aberto e consequente incremento de contato entre as águas).

$\mathrm{Na}$ porção inferior do poço $\mathrm{BO}$ destaca-se a superdominância de palinoforaminíferos trocoespirais entre as profundidades $2.690,5 \mathrm{~m}$ e $2.691,5 \mathrm{~m}$, onde a curva de dominância atinge o valor 0,7 devido à sua predominância na associação de palinomorfos marinhos. Esse fato condiz com o panorama vigente antes da transgressão albiana, momento em que as condições ambientais eram estressantes: mar restrito com baixa oxigenação e baixo teor de nutrientes (ARTHUR \& SCHLANGER 1979; DIAS-BRITO 1987, 1995). É provável que, nesse momento, os foraminíferos tenham sido os representantes da microfauna marinha que melhor toleravam as condições adversas em que estavam inseridos e, assim, obtiveram grande sucesso reprodutivo e ocuparam os nichos vagos.

A biogeografia das espécies de dinoflagelados encontradas nos poços serve de indicativo para explicar como ocorreu a entrada das águas durante a formação do Atlântico Sul. A ausência de dinoflagelados próprios de águas austrais (Ascodinium acrophorum, Occisucysta victorii e Tenua americana) e a presença de dinoflagelados tipicamente tetianos no material estudado (Codoniella campanulata, Cyclonephelium vannophorum, Endoceratium dettmanniae e Tehamadinium mazaganense) permitem inferir que durante o Albiano, a Bacia de Campos apresentava 


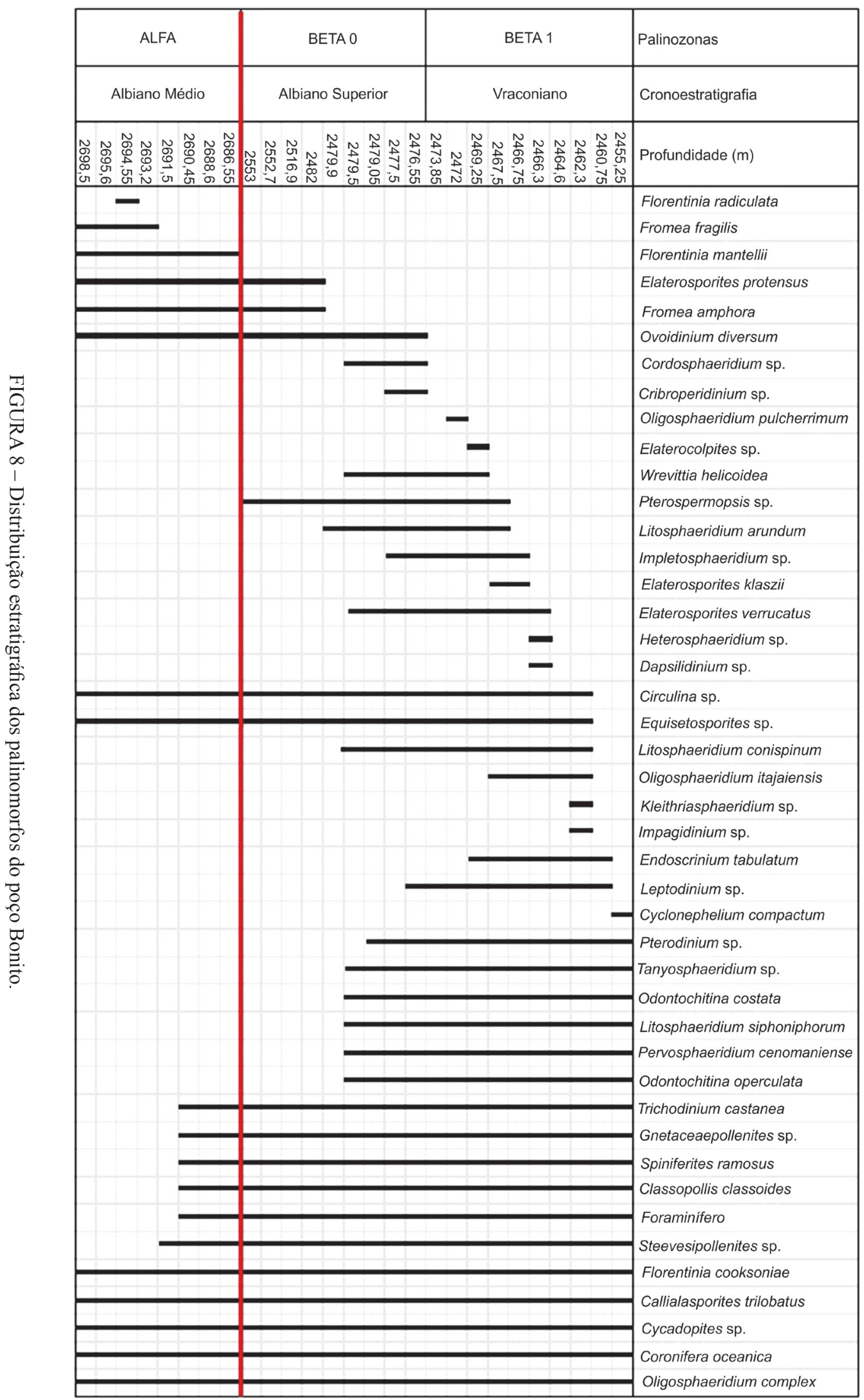




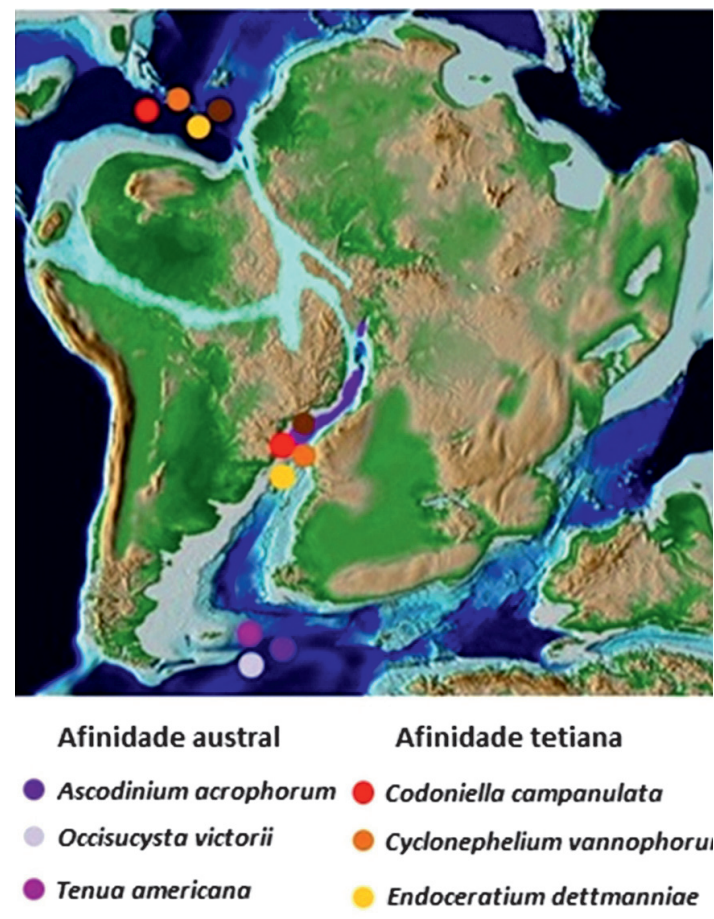

FIGURA 9 - Espécies de dinoflagelados típicas das microfloras austral (representadas em cores frias) e tetiana (representadas em cores quentes).

biota com forte caráter tetiano, fato que se opõe ao modelo tradicional de influência austral no início de formação do Atlântico Sul, defendido por BOLLI (1978) (Figura 9). Tendo como base a integração dos dados micropaleontológicos citados na introdução desse artigo e, em especial, os dados referentes à associação de dinoflagelados do Albiano inferior, é possível confirmar a teoria de ARAI (2009) e inferir que a entrada de águas para o estabelecimento do Atlântico Sul não obedeceu à abertura tectônica de sul para norte, em função da existência de uma barreira física que impedia a livre circulação oceânica - o microcontinente Rio Grande.

Dessa forma, durante a elevação eustática do Aptiano, as águas provenientes do Atlântico Central transbordaram e invadiram as margens equatorial e leste da América do Sul, estabelecendo o início do Atlântico Sul primitivo com forte influência do Mar de Tétis, condição esta que prevaleceu praticamente no decorrer do Albiano.

Apenas no final do Albiano (Vraconiano) é que a diversidade da biota marinha aumenta consideravelmente em um ambiente francamente oceânico; muitas espécies de dinoflagelados passam a ser encontradas no registro sedimentar, entre elas espécies típicas de mares austrais, o que atesta o desaparecimento de barreira efetiva.

\section{CONCLUSÕES}

O estudo integrado dos palinomorfos da Bacia de Campos, em especial a assembleia de dinoflagelados, mostrou-se uma ferramenta útil para as interpretações paleoambientais e paleobiogeográficas do Cretáceo Inferior, em particular do Albiano. Os resultados apresentados neste artigo fornecem maior embasamento para futuras pesquisas de cunho palinológico e permitem as seguintes conclusões:

- Os palinomorfos continentais confirmam que o clima durante o Albiano no Brasil era quente e seco e a cobertura vegetal próxima à Bacia de Campos era dominada por Gimnospermas arbóreas e herbáceas. Havia presença pontual de pteridófitas resistentes à aridez.

- A comparação entre a distribuição de elementos marinhos e continentais permite inferir que a Bacia de Campos, por ocasião da sedimentação da Formação Macaé, era caracterizada por ambiente marinho, tendo em vista a predominância de elementos como dinoflagelados e palinoforaminíferos.

- No entanto, havia influxo constante de material terrígeno, fato este atestado pela presença subordinada de palinomorfos continentais (esporos e grãos de pólen) em todas as amostras. Mesmo com o processo de estabelecimento do Oceano Atlântico Sul e o espessamento da coluna d'água, os palinomorfos continentais se fazem presentes no registro sedimentar e são encontrados em maior quantidade no final do Albiano, quando a litologia passa gradativamente de carbonatos para folhelhos e margas. Dessa forma, é possível afirmar que o aporte de material terrígeno para dentro da bacia aumentou no final do Albiano.

- Apesar da maioria das espécies de dinoflagelados encontrada ser cosmopolita, algumas espécies provincialistas foram encontradas e trazem consigo informações biogeográficas relevantes. A presença de espécies de dinoflagelados tetianos e a ausência de dinoflagelados austrais no Albiano da Bacia de Campos - em adição a dados micropaleontológicos anteriores oriundos de palinoforaminíferos, nanofósseis e amonitas - possibilitam inferir que a entrada de águas durante o estabelecimento do Oceano Atlântico Sul ocorreu de Norte para Sul.

\section{AGRADECIMENTOS}

Os autores agradecem toda equipe do UNESPetro - Centro de Geociências Aplicadas ao Petróleo, Rio Claro - pelo suporte técnico necessário para a realização da pesquisa. Agradecem também à PETROBRAS pelo financiamento do trabalho e pela concessão do material utilizado. E, finalmen- 
te, agradecimentos destinados aos editores e relatores da Revista do Instituto Geológico pela revisão crítica do texto.

\section{REFERÊNCIAS BIBLIOGRÁFICAS}

ARAI, M. 2009. Paleogeografia do Atlântico Sul no Aptiano: um novo modelo a partir de dados micropaleontológicos recentes. Boletim de Geociências da Petrobrás, 17(2): 331-351.

ARAI, M.; LANA, C.C. 2004. Histórico do estudo de dinoflagelados fósseis no Brasil: sua relação com a evolução da exploração petrolífera no Cretáceo das bacias da margem continental. Boletim de Geociências da Petrobras, 12(1): 175-189.

ARAI, M.; UESUGUI, N. 1989. Dinoflagellate biostratigraphy of the Macaé Formation (Albian-Cenomanian), Campos Basin. In: INTERNATIONAL CONFERENCE ON MODERN AND FOSSIL DINOFLAGELLATES, 4, Woods Hole, Program and Abstracts, p. 20.

ARAI, M.; LANA, C.C.; PEDRÃO, E. 1994. Ecozona Subtilisphaera spp.: Registro eocretáceo de um importante episódio ecológico do Oceano Atlântico primitivo. Acta Geológica Leopondensia, XVII, 39(2): 521-538.

ARAI, M.; BOTELHO NETO, J.; LANA, C.C.; PEDRÃO, E. 2000. Cretaceous dinoflagellate provincialism in Brazilian marginal basins. Cretaceous Research, 21: 351-366.

ARTHUR, M.A.; SCHLANGER, S.O. 1979. Cretaceous oceanic anoxic events as causal factors in development of reef reservoired giant oil fields. AAPG Bulletin, 63(6): 870-885.

AURISANO, R.W. 1992. Palynostratigraphy, graphic correlation and the Albian- Cenomanian boundary offshore Gabon and Congo. Bulletin Des Centres Recherches Exploration Production Elf Aquitaine, 63: 347-363.

BENGTSON， P.; KOUTSOUKOS， E.A.M.; KAKABADZE, M.V. 2007. Ammonite and foraminiferal biogeography and the opening of the Equatorial Atlantic gateway. $\quad I n$ : INTERNATIONAL PALAEOBIOGEOGRAPHY SYPOSIUM, 1 , Paris, Abstracts, p. 12.

BOLLI, H. M. 1978. Cretaceous and Paleogene calcisphaerulidae from DSDP Leg 40, southeastern Atlantic. In: H.M.Bolli \& W.E.F. Ryan (ed.) Initial Reports of the Deep Sea Drilling Project. Washington, Government Printing Office, 40: 819-837.

CHANG, Y. M. 1967. Accuracy of fossil percentage estimation. Journal of Paleontology, 41(2): 500-502.

COSTA, L.I.; DAVEY, R.J. 1992. Dinoflagellate cysts of the Cretaceous System. In: A.J. Powell (ed.) A Stratigraphic Index of Dinoflagellate Cysts. London, Chapman \& Hall, p. 99-153.

DAVEY, R.J. 1979. Marine Apto-Albian palynomorphs from Holes 400A and 402A, IPOD Leg 48, northern Bay of Biscay. In: L. Montadert (ed.). Deep Sea Drilling Project. Washington, Initial Reports, 48: 547-577.

DIAS, J.L. 2005. Tectônica, estratigrafia e sedimentação no Andar Aptiano da margem leste brasileira. Boletim de Geociências da Petrobras, 13(1): 7-25.

DIAS, J.L. 2008. Estratigrafia e sedimentação dos evaporitos neo-aptianos na margem leste brasileira. In: W. Mohriak, P. Szatmari \& S.M. Couto Anjos (eds.) Sal- Geologia e tectônica: exemplos nas bacias brasileiras. Rio de Janeiro, Petrobras/Beca Edições Ltda., p. 220-229.

DIAS-BRITO, D. 1982. Evolução Paleoecológica da Bacia de Campos durante a deposição dos calcilutitos, margas e folhelhos da Formação Macaé (Albiano e Cenomaniano?). Boletim Técnico da Petrobrás, 25(2): 84-97.

DIAS-BRITO, D. 1987. A Bacia de Campos no Mesocretáceo: uma contribuição à Paleoceanografia do Atlântico Sul primitivo. Revista Brasileira de Geociências, 17(2): 162-167.

DIAS-BRITO, D. 1995. Calcisferas e microfácies em rochas carbonáticas pelágicas mesocretáceas. Universidade Federal do Rio Grande do Sul, Porto Alegre, Tese de Doutorado, 688 p.

DIAS-BRITO, D. 2000. Global stratigraphy, palaeobiogeography and palaeoecology of Albian-Maastrichtian pithonellid calcispheres: impact on Tethys configuration. Cretaceous Research, 21: 315-349.

DINO, R.; POCKNALL, D.T.; DETTMANN, M.E. 1999. Morphology and ultrastructure of elater-bearing pollen from the Albian to Cenomanian of Brazil and Ecuador: 
implications for botanical affinity. Review of Palaeobotany and Palynology, 105: 201-235.

FENSOME, R.A.; MACRAE, R.A.; WILLIAMS, G.L. 2008. DINOFLAJ2, Version 1. American Association of Stratigraphic Palynologists, Data Series no. 1. Disponível em http:// dinoflaj.smu.ca/wiki/Main_Page. Acessado em 12 mai. 2015.

HAMMER, Ø.; HARPER, D.A.T.; RYAN, P.D. 2001. PAST: Paleontological Statistics Software Package for Education and Data Analysis. Paleontologia Electronica, 4(1): 9 p.

JUGGINS, S. 2007. C2 Version 1.5 User Guide. Software for ecological and palaeoecological data analysis and visualization. Newcastle University, Newcastle upon Tyne, UK. 73p.

KENNEDY, W.J.; COOPER, M. 1975. Cretaceous ammonite distributions and the opening of the South Atlantic. Journal of the Geological Society, 131(3): 283-288.

KOUTSOUKOS, E.A.M.; DIAS-BRITO, D. 1987. Paleobatimetria da margem continental do Brasil durante o Albiano. Revista Brasileira de Geociências, 17(2): 86- 91.

LARSON, R. L.; LADD, J.W. 1973. Evidence for the opening of the South Atlantic in the Early Cretaceous. Nature, 246: 209-212.

LENTIN, J.K.; WILLIAMS, G.L. 2004. The Lentin and Williams index of fossil dinoflagellates. 2004 edition. American Association of Stratigraphic Palynologists Foundation, 42: 909 p.

OJEDA, H.A.O. 1982. Structural framework, stratigraphy, and evolution of Brazilian Marginal Basins. The American Association of Petroleum Geologists Bulletin, 6(6): 732-749.
OOSTING, A.M.; LEEREVELD, H.; DICKENS, G.R.; HENDERSON, R.A.; BRINKHUIS, H. 2006. Correlation of Barremian-Aptian (midCretaceous) dinoflagellate cyst assemblages between the Tethyan and Austral realms. Cretaceous Research, 27: 792-813.

RANGEL, H.D.; MARTINS, F.A.L.; ESTEVES, F.R.; FEIJÓ, F.J. 1994. Bacia de Campos. Boletim de Geociências da Petrobrás. Rio de Janeiro. 8(1): 203-217.

SCHALLER, H. 1973. Estratigrafia da Bacia de Campos. In: SBG, CONGRESSO BRASILEIRO DE GEOLOGIA, 27, Aracaju. SIMPÓSIO SOBRE EXPLORAÇÃO DE PETRÓLEO NO BRASIL, 3, p. 247-258.

TORRICELLI, S. 2000. Lower Cretaceous dinoflagellate cyst and acritarch stratigraphy of the Cismon APTICORE (Southern Alps, Italy). Review of Paleobotany and Palynology, 108: 213-266.

UESUGUI, N. 1979. Palinologia: Técnicas de tratamento de amostras. Boletim Técnico PETROBRAS, 22(4): 229-240.

WIEDMANN, J.; NEUGEBAUER, J. 1978. Lower Cretaceous Ammonites from the South Atlantic Leg 40 (DSDP), their stratigraphic value and sedimentological properties. In: H.M. Bolli \& W.B.F. Ryan (ed.). Init. Repts. DSDP. Washington, U. S. Govt. Print Office, p. 709-734.

WILLIAMS, G.L.; BUJAK, J.P. 1985. Mesozoic and Cenozoic dinoflagellates. In: H.M. Bolli; J.B. Saunders \& K. Perch-Nielsen (ed.) Plankton Stratigraphy. Cambridge University Press, p. 847-964.

WINTER, W.R.; JAHNERT, R.J.; FRANÇA, A.B. 2007. Bacia de Campos. Boletim de Geociências da Petrobras. Rio de Janeiro, 15(2): 511-529.

\section{Endereço dos autores:}

Clara Rodrigues Nascimento e Mitsuru Arai - UNESPetro, Centro de Geociências Aplicadas ao Petróleo, Instituto de Geociências e Ciências Exatas, Universidade Estadual Paulista Júlio de Mesquita Filho, Avenida 24 A, 1515, 13506-900, Rio Claro, SP, Brasil.E-mails: claranasc@hotmail.com, mitsuru.arai@ gmail.com

José Alexandre de Jesus Perinotto - Instituto de Geociências e Ciências Exatas, Universidade Estadual Paulista Júlio de Mesquita Filho, Departamento de Geologia Aplicada, Avenida 24 A, 1515, 13506-900, Rio Claro, SP, Brasil.E-mail: perinoto@rc.unesp.br

Artigo submetido em 27 de agosto de 2015, aceito em 4 de novembro de 2015. 


\section{APÊNDICE \\ LISTA DOS TÁXONS IDENTIFICADOS}

\section{ESPOROS}

Cicatricosisporites Potonié \& Gelletich, 1933 - Figura 2A

\section{GRÃOS DE PÓLEN}

Callialasporites trilobatus (Balme) Dev, 1962 - Figura 2F

Classopolis classoides Pflug, 1953 - Figura 2D,E

Circulina Malyawkina, 1949 - Figura 2B

Cycadopites Wodehouse, 1933 ex Wilson \& Webster, 1946 - Figura 2C

Elaterocolpites castelainii Jardiné \& Magloire, 1965

Elaterosporites klaszii (Jardiné \& Magloire) Jardiné, 1967 - Figura 2G

Elaterosporites protensus (Stover) Jardiné, 1967 - Figura 2H

Elaterosporites verrucatus Jardiné, 1967 - Figura 2I

Equisetosporites (Daugherty, 1941) emend. Singh, 1964 - Figura 2J,K

Gnetaceaepollenites Thiegart, 1938 - Figura 2L,M

Steevesipollenites Stover, 1964 - Figura 2N

\section{DINOFLAGELADOS}

Achomosphaera Evitt, 1963

Adnatosphaeridium Williams \& Downie, 1966

Atopodinium iuvene Prössl, 1990

Atopodinium mirabile (Below, 1984) Masure, 1991

Batiacasphaera Drugg, 1970

Caligodinium aceras (Manum \& Cookson, 1964) Lentin \& Williams, 1973

Circulodinium distinctum distinctum Deflandre \& Cookson, 1955

Circulodinium distinctum longispinatum (Davey, 1978) Lentin \& Williams, 1989

Circulodinium paucispinum (Davey, 1969) Fauconnier \& Masure, 2004

Codoniella campanulata (Cookson \& Eisenack, 1960) Downie \& Sarjeant, 1965

Cometodinium sp. aff. C. obscurum Deflandre \& Courteville, 1939

Cordosphaeridium Eisenack, 1963 - Figura 3F

Coronifera oceanica Cookson \& Eisenack, 1958 - Figura 3D

Craspedodinium indistinctum Cookson \& Eisenack, 1974

Cribroperidinium Neale \& Sarjeant, 1962 - Figura 4A,B

Cribroperidinium edwardsii (Cookson \& Eisenack, 1959) Davey, 1969

Cribroperidinium fetchamense (Sarjeant, 1966) Helenes, 1984

Cribroperidinium tensiftense Below, 1981

Cyclonephelium compactum Defalndre \& Cookson, 1955

Cyclonephelium paucimarginatum Cookson \& Eisenack, 1962

Cyclonephelium vannophorum Davey, 1969 - Figura 3E

Dapsilidinium laminaspinosum (Davey \& Williams, 1966) Lentin \& Williams, 1981

Dinopterygium cladoides Deflandre, 1935

Downiesphaeridium flexuosum (Davey et al., 1966) Islam, 1993

Endoceratium dettmanniae (Cookson \& Hughes, 1964) Stover \& Evitt, 1978

Endoscrinium tabulatum Miles, 1990

Escharisphaeridia Erkmen \& Sarjeant, 1980

Florentinia Davey \& Verdier, 1973 - Figura 3G

Florentinia cooksoniae Singh, 1971

Florentinia ferox (Deflandre, 1937) Duxbury, 1980

Florentinia mantellii (Davey \& Williams, 1966) Davey \& Verdier, 1973 
Florentinia radiculata (Davey \& Williams, 1966) Davey \& Verdier, 1973

Fromea amphora Cookson \& Eisenack, 1958 - Figura 4C

Fromea fragilis (Cookson \& Eisenack, 1962) Stover \& Evitt, 1978

Fromea quadrugata Duxbury, 1980

Gordiacysta coronata Miles, 1990

Hapsocysta peridictya (Eisenack \& Cookson, 1960) Davey, 1979

Heterosphaeridium heteracanthum (Deflandre \& Cookson, 1955) Eisenack \& Kjellstrom, 1972

Hystrichodinium pulchrum Deflandre, 1935

Impagidinium Stover \& Evitt, 1978

Impletosphaeridium Morgenroth, 1966 - Figura 3H

Kallosphaeridium ringnesiorum (Manum \& Cookson, 1964) Helby, 1987

Kallosphaeridium romaense (Burger, 1980) Burger, 1980

Kiokansium polypes (Cookson \& Eisenack, 1962) Below, 1982

Kleithriasphaeridium Davey, 1974

Leberidocysta chlamydata (Cookson \& Eisenack, 1962) Stover \& Evitt, 1978

Leptodinium Klement, 1960 - Figura 4D

Litosphaeridium arundum Eisenack \& Cookson, 1960

Litosphaeridium conispinum Davey \& Verdier, 1973 - Figura 3A, B

Litosphaeridium siphoniphorum Cookson \& Eisenack, 1958

Muderongia sp. cf. M. pariata Duxbury, 1983

Odontochitina ancala Bint, 1986

Odontochitina costata Alberti, 1961

Odontochitina sp. aff. O. cribropoda Deflandre \& Cookson, 1955

Odontochitina operculata Wetzel, 1933 - Figura 4E,F

Odontochitina rhakodes Bint, 1986

Odontochitina singhii Morgan, 1980

Oligosphaeridium complex (White, 1842)

Oligosphaeridium itajaiensis Masure \& Arai, 2003 - Figura 3I

Oligosphaeridium pulcherrimum Deflandre \& Cookson, 1955

Oligosphaeridium totum Brideaux, 1971

Ovoidinium diversum Davey, 1979

Palaeohystrichophora infusorioides Deflandre, 1935

Palaeoperidinium cretaceum (Pocock, 1962) Lentin \& Williams, 1976

Pervosphaeridium cenomaniense Norvick, 1976 - Figura 4G

Pervosphaeridium truncatum (Davey, 1969) Below, 1982

Prolixosphaeridium Davey et al., 1996 - Figura 3M

Prolixosphaeridium conulum Davey, 1969

Pseudoceratium Gocht, 1957 - Figura 4H

Pterodinium cingulatum cingulatum Wetzel, 1933

Spiniferites ramosus (Ehrenberg, 1838) Davey \& Williams, 1966 - Figura 3J

Spiniferites bejui Masure et al., 1998

Tanyosphaeridium Davey \& Williams, 1966 - Figura 3K

Tanyosphaeridium isocalamum (Deflandre \& Cookson, 1955) Davey \& Williams, 1969

Tanyosphaeridium variecalamum Davey \& Williams, 1966

Tehamadinium mazaganense (Below, 1984) Jan Du Chene et al., 1986

Trichodinium castanea Deflandre, 1935 - Figura 3L

Walvisia woodii Miles, 1990

Wrevittia helicoidea (Eisenack \& Cookson, 1960) Helenes \& Lucas-Clark, 1997 - Figura 4I

Xiphophoridium alatum (Cookson \& Eisenack, 1962) Sarjeant, 1966

\section{DEMAIS MARINHOS}

Pterospermopsis Wetzel, 1952 - Figura 4J

Escolecodonte - Figura 4K

Palinoforaminífero espiral - Figura 4L 